\title{
Effet de la fertilisation minérale sur la culture du riz (Oryza sativa L.) et du blé dur (Triticum durum Desf.) dans la vallée du fleuve Sénégal
}

\author{
Habibou Gueye ${ }^{1, *}$, Amadou T. Sall ${ }^{2,}$ Boubou G. Kéita ${ }^{1}$, Saliou N'Diaye ${ }^{3}$, Hamidou Dieng, Tal- \\ la Gueye ${ }^{3}$. \\ ${ }_{1}$ Centre national de recherche agronomique et de développement agricole (CNARDA), Kaédi 40101, Mauritanie ; \\ 2Institut Sénégalais de Recherches Agricoles (ISRA), Saint-Louis 46024, Sénégal; tidianesall11@yaboo.com \\ ${ }^{3}$ Ecole Nationale Supérieure d'Agriculture (ENSA), Université de Thiès, Sénégal. BP A 296 Thiès, Sénégal; drsa- \\ liou@gmail.com; saliou.ndiaye@univ-thies.sn,tgueve@univ-tbies.sn; \\ ${ }_{4}^{4}$ Université de Nouakchott AL Aasriya (UNA). Nouakchott. B.P. 5026 Nouakchott. R.I. Mauritanie ; dihamidou@yahoo.fr \\ * Corresponding author: gueye habibou@yaboo.fr ; Tel: +22246718431
}

Mots clés : Fertilisation raisonnée, riz, blé dur, rotation culturale, vallée du Fleuve Sénégal

Key words: rational fertilization, rice, durum wheat, crop rotation, Senegal River Valley

Publication date 31/07/2019, http://www.m.elewa.org/JAPS

\section{RÉSUMÉ}

Le travail a pour objectif de déterminer les combinaisons d'élément nutritif $\mathbf{N}$, $\mathbf{P}$ et $\mathrm{K}$ optimal pour améliorer la productivité et la rentabilité du riz et du blé dans la vallée du fleuve Sénégal. Des expérimentations ont été conduites au niveau des stations expérimentales du Centre $\mathrm{Na}$ tional de Recherche Agronomique et de Développement Agricole (CNRADA) de la Mauritanie à Sylla dans la région du Gorgol et à Dara Salam dans la région du Trarza. Des parcelles comparatives ont été mises en place pendant les saisons 2016-2017 et 2017-2018 dans un dispositif expérimental en bloc de Fisher pour mesurer les effets de différents apports de fertilisation minérale sur la performance du riz et du blé. Un calendrier cultural visant à faire trois saisons de cultures par an a été établie avec deux saisons de riz et une saison de blé. Les résultats montrent que pour le riz, si l'application de $\mathbf{N}$ est nécessaire pour augmenter la production de chaque saison, ce n'est probablement pas le cas pour P et K. Par exemple, les applications saisonnières de $\mathbf{N}$ (chaque saison de culture) et des applications annuelles (une saison par an) de $P$ et de $K$ pourraient être une option pour améliorer la gestion des engrais, la production et rentabilité du riz. Par contre la culture du blé nécessite l'application saisonnière de $\mathbf{N}, \mathbf{P}$ et $\mathrm{K}$ pour optimiser la production.

\section{ABSTRACT}

The objective of the work is to determine the optimal $\mathrm{N}, \mathrm{P}$ and $\mathrm{K}$ nutrient combinations to improve the productivity and profitability of rice and wheat in the Senegal River Valley. Experiments were conducted at the experimental stations of the Center for Agronomic Research and Agricultural Development (CNRADA) of Mauritania at Sylla in the Gorgol region and at Dara in the region of Trarza. Comparative plots were set up during the 2016-2017 and 2017-2018 seasons to measure the effects of different mineral fertilizer inputs on the performance of rice and wheat. A crop calendar for three growing seasons per year has been established with two seasons of rice and one season of wheat. The results show that for rice, if the application of $\mathbf{N}$ is necessary to increase the production of each season, this is probably not the case for $\mathbf{P}$ and $K$. For example, the seasonal applications of $\mathbf{N}$ (each growing season) and annual applications 
(one season per year) of $\mathbf{P}$ and $\mathrm{K}$ could be an option to improve fertilizer management, rice production and profitability. On the other hand, wheat cultivation requires the seasonal application of $\mathbf{N}, \mathbf{P}$ and $\mathrm{K}$ to optimize production.

\section{INTRODUCTION}

Le riz et le blé sont les deux céréales les plus importantes dans le monde. Ces deux céréales sont cultivées en rotation au Bangladesh, en Inde et au Pakistan (Ladha et al. 2000). Dans la vallée du fleuve Sénégal, environ 120000 ha sont intensivement cultivés avec la double culture du riz (FAO, 1997) pendant les deux saisons chaudes et la saison froide entre mi-novembre et début mars ne convenant pas à la culture du riz (Dingkuhn et Sow, 1997), les champs sont pour la plupart laissés en jachère. Les travaux de recherches dans la vallée ont montré que des variétés tolérantes à la chaleur pourraient être cultivées pendant cette période (Sall et al., 2018). Puisque la plupart des agriculteurs de la région dépendent de leurs champs pour leur fournir de la nourriture, la rotation des cultures ne peut être pratiquée de manière réaliste qu'entre cultures de base (riz-blé). Cependant le développement d'un système de culture riz-riz-blé va entrainer la dégradation des sols et l'appauvrissement en éléments nutritifs nécessitant l'utilisation d'une fertilisation raisonnée pour une agriculture durable. La durabilité

\section{MATÉRIEL ET MÉTHODES}

\subsection{Site d'implantation de l'essai et matériel} végétal: Les expérimentations ont été conduites dans la vallée du Fleuve Sénégal au niveau des stations expérimentales du Centre National de Recherche Agronomique et de Développement Agricole (CNRADA) de la Mauritanie à Sylla dans la région du Gorgol $\left(16^{\circ} 09^{\prime} ; 13^{\circ} 30^{\prime}\right)$ et à Dara Salam dans la région du Trarza $\left(16^{\circ} 31^{\prime} ; 15^{\circ} 48^{\prime}\right)$. A Dara on a un sol brun jaunâtre à blanc sableux, avec quelques poches argileuses peu compact poreux de texture argilolimoneux-sablonneux avec une teneur en matière organique élevée et une bonne capacité de rétention d'eau, tandis qu'à Sylla les sols sont de couleur brun olive avec différenciation de couches plus légères sablo-limoneux avec une capacité de rétention d'eau intermédiaire. Tous les sols sont riches en phosphore $(\mathrm{P})$ et pauvres en autres éléments nutritifs, ce qui est typique de l'effet "Sahara » (Boy et al., 2008). Un calendrier cultural visant à faire trois saisons de cul- implique que des rendements élevés soient maintenus mais aussi que les pratiques agricoles soit acceptables pour des impacts environnementaux à long terme (Tilman et al., 2002). Pour les pays en développement, les engrais chimiques représentent une dépense importante et donc pour chaque culture, les agronomes doivent être en mesure de déterminer la quantité et le type d'élément nutritif les plus appropriés pour améliorer la productivité et la rentabilité du riz et du blé dans les zones irriguées. C'est dans ce sens qu'intervient cette étude afin de développer un système de culture combinant les deux cultures (riz et blé) pour intensifier la production agricole et réponde à la demande croissante des populations. Ceci n'est possible qu'en cherchant à mieux intégrer les moyens d'améliorer la production de la culture du riz et celle du blé dans la vallée du fleuve Sénégal dans le processus cultural déjà existant et en proposant de meilleures combinaisons possibles pour une fertilisation minérale raisonnée et durable.

tures par an a été établie avec deux saisons de riz et une saison de blé. Pour le riz, deux variétés à cycle court (Sahel 108 et Sahel 305) ne dépassant pas 120 jours en contre saison froide-chaude et largement vulgarisées dans la vallée du fleuve Sénégal sont utilisé tandis que la variété de blé dur Karim adaptée aux conditions agro climatiques de la vallée pendant la saison froide est utilisée. Cette variété d'un cycle de 90 jours est souvent utilisée en rotation avec le riz. L'utilisation des variétés de riz et de blé à cycle court permettent aux paysans d'utiliser les rizières pendant toutes les saisons sans chevauchement de campagnes. Ceci a été expérimenté dans cette étude à Sylla en cultivant le riz pendant la contre saison chaude qui dure de Mars à Juin et pendant la saison des pluies qui s'étend de Juillet à Octobre puis le blé a été cultivé en rotation pendant la saison froide de Novembre à Février. 
3.2 Facteur étudié et dispositif expérimental : Le facteur dose de fumure minérale a été étudié dans les essais avec cinq niveaux de fertilisation qui constituent les traitements des essais appliqués au stade tallage, montaison et initiation paniculaire.

- $\quad$ T1 (Témoin absolu) = aucune application d'engrais

$$
\begin{array}{ll}
- & \mathrm{T} 2(\mathrm{~N})=120 \mathrm{~kg} \mathrm{~N} \mathrm{ha}^{-1} \\
\text { - } & \mathrm{T} 3(\mathrm{NP})=120 \mathrm{~kg} \mathrm{~N} \mathrm{ha}{ }^{-1}+26 \mathrm{~kg} \mathrm{Pha} \\
\text { - } & \text { T4 }(\mathrm{PK})=26 \mathrm{~kg} \mathrm{Pha}^{-1}+50 \mathrm{~kg} \mathrm{~K} \mathrm{ha}^{-1} \\
\text { T5 }(\mathrm{NPK})=120 \mathrm{~kg} \mathrm{~N} \mathrm{ha}^{-1}+26 \mathrm{~kg} \mathrm{P} \mathrm{ha}^{-1}+
\end{array}
$$$$
50 \mathrm{~kg} \mathrm{~K} \mathrm{ha-1}
$$

Des parcelles comparatives ont été mises en place pendant les saisons 2016-2017 et 2017-2018 pour

\section{$4 \quad$ RÉSULTATS}

4.1 Effet de la fertilisation minérale sur la performance du riz: L'analyse de variance montre que la performance des deux variétés de riz utilisées n'est pas significativement différente du point de vue statistique aussi bien pour le rendement grain que la biomasse aérienne et l'indice de récolte. Les deux variétés de riz se comportent de la même façon par rapport aux différentes combinaisons de fumure. Autrement dit, les trois caractères agronomiques mesurer les effets des apports de fertilisation minérale sur le riz et le blé. Pour le riz le facteur fumure a fait l'objet d'étude sur deux variétés différentes pendant deux saisons successives au niveau de la station de Sylla tandis que pour le blé l'étude s'est portée sur une seule variété au niveau des deux stations avec deux campagnes différentes. Pour chacune des campagnes et au niveau de chaque site, le dispositif expérimental utilisé est en bloc de Fischer avec 4 répétitions. Des paramètres de rendements grain (Rdt), biomasse aérienne (Biom) et indice de récolte (IR) ont été évalués pour chaque essai et des analyses de variances et des tests de comparaison multiple effectuée.

(Rdt, Biom et IR) étudiés ne varient pas d'une variété à l'autre suivant les différentes fumures. Toutefois, il existe une différence significative entre les cinq niveaux de fumure sur la performance des variétés de riz avec un seuil de significativité $(\operatorname{Pr}<0.05)$ pour le rendement grain et l'indice de récolte et un seuil de significativité $(\operatorname{Pr}<0.01)$ pour la biomasse aérienne (Tableau 1).

Tableau 1: Analyse globale des Effets de la fertilisation sur les variétés de riz

\begin{tabular}{l|l|l|l|l|l|l|l}
\hline & \multirow{2}{*}{} & \multicolumn{2}{|l|}{ Rendement grain } & \multicolumn{2}{l|}{ Biomasse aérienne } & \multicolumn{2}{l}{ Indice de récolte } \\
\cline { 3 - 8 } & Df & Sum Sq & $\operatorname{Pr}(>\mathbf{F})$ & Sum Sq. & $\operatorname{Pr}(>\mathbf{F})$ & Sum Sq. & $\operatorname{Pr}(>\mathbf{F})$ \\
\hline Block & 3 & 1864627 & $0.0213^{*}$ & 1993039 & 0.7990 & 144.6 & $0.0019^{* *}$ \\
Génotype & 1 & 125552 & 0.3881 & 469156 & 0.6298 & 4.9 & 0.4247 \\
Fumure & 4 & 2351205 & $0.0177^{*}$ & $4.2 \mathrm{E}+07$ & $0.0026^{* *}$ & 81.85 & $0.0491^{*}$ \\
Génotype : Fumure & 4 & 562256 & 0.4995 & 6752061 & 0.5031 & 22.85 & 0.5568 \\
Résiduels & 27 & 4405204 & & $5.3 \mathrm{E}+07$ & & 201.4 & \\
\hline
\end{tabular}

*significativité à un seuil $(\operatorname{Pr}<0.05)$

**significativité à un seuil $(\operatorname{Pr}<0.005)$

Le rendement moyen des deux variétés de riz est de $1902 \mathrm{~kg} \mathrm{ha}^{-1}$ sous les différents traitements de fumure alors que la moyenne de la biomasse aérienne est de $10079 \mathrm{~kg} \mathrm{ha}{ }^{-1}$. La variation du rendement grain pour chaque variété au niveau des différents traitements est présentée à la figure 1. Puisque la réponse des deux variétés par rapport aux traitements n'est pas significativement différente nous considérons l'effet global de chaque traitement sur les deux variétés de riz. En effet les traitements T2, T3 et T5 ont des effets sur le rendement significativement différent du traitement T1 (Tableau 2). De même pour la production de la biomasse aérienne le traitement T3 a un effet supérieur et significativement différent de tous les autres traitements tandis que cette différence significative n'est observée qu'entre les traitements T5 et T1 (Tableau 2). 


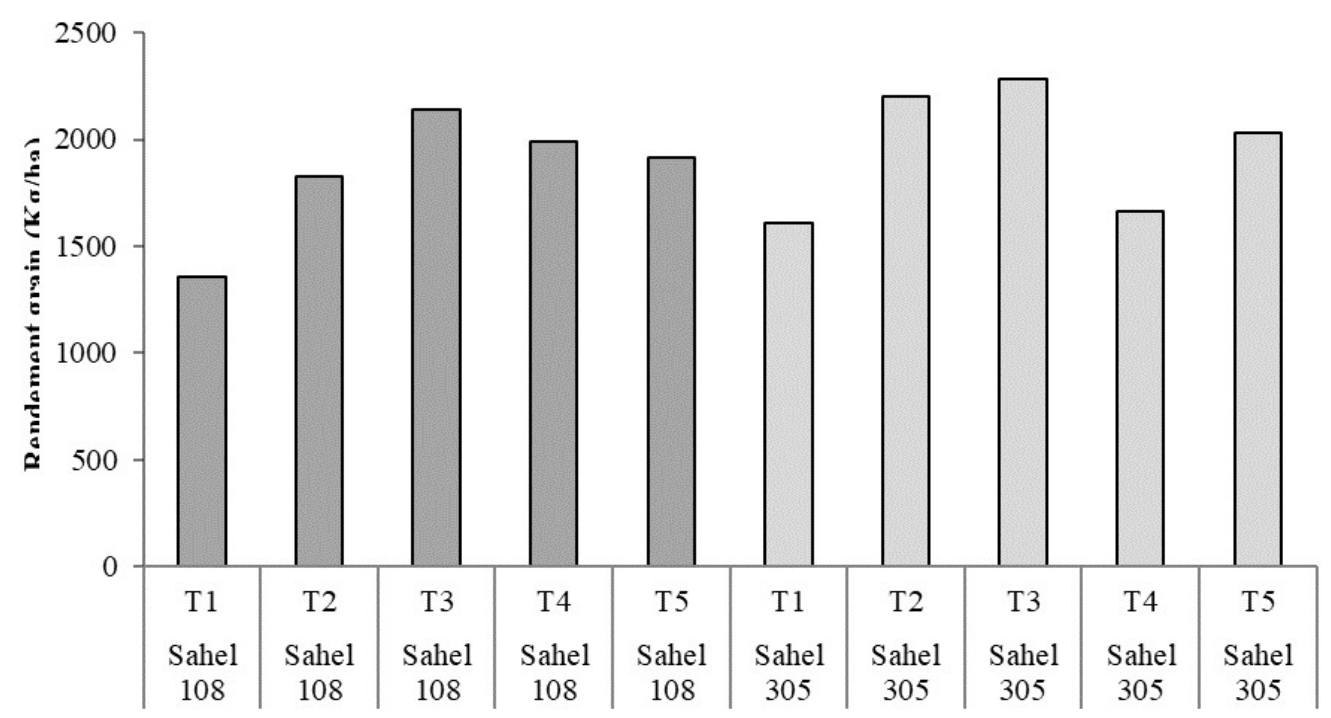

Figure 1 : Effets des différents niveaux de fertilisation minérale sur le rendement de deux variétés de riz

Tableau 2 : Test de comparaison multiple de différents traitements de fumure sur la performance du riz

\begin{tabular}{l|c|c}
\hline Fumure & Rendement & Biomasse aérienne \\
\hline T1 & $1483.8 \mathrm{~d}$ & $8791.6 \mathrm{~cd}$ \\
T2 & $2014.5 \mathrm{ab}$ & $9531.1 \mathrm{bcd}$ \\
T3 & $2211.3 \mathrm{a}$ & $11895.8 \mathrm{a}$ \\
T4 & $1828.4 \mathrm{abcd}$ & $9937.5 \mathrm{bc}$ \\
T5 & $1974.5 \mathrm{abc}$ & $10239.5 \mathrm{~b}$ \\
PPDS & 414.4 & 1441.3 \\
\hline
\end{tabular}

$N B$ : Deux traitements ayant une lettre en commun ne sont pas significativement différents

4.2 Effet de la fertilisation minérale sur la performance du blé : La réponse de différents apports de fertilisation minérale sur une variété de blé a été observée au niveau des deux stations. L'analyse de variance des deux environnements combinés montre que l'effet des différents traitements sur le rendement et la biomasse n'est pas significativement différent entre les traitements eux même et entres les environnements. La différence significative entre les traitements eux même et entres les environnements est notée seulement pour l'indice de récolte (Tableau 3). Il existe une importante interaction entre les traitements et les différents environnements concernant l'indice de récolte.

Tableau 3 : L'analyse de variance des effets de traitements des deux campagnes combinées sur le rendement du blé, la biomasse aérienne et l'indice de récolte.

\begin{tabular}{l|l|l|l|l|l|l|l|l}
\hline & \multicolumn{3}{|l|}{} & \multicolumn{2}{l|}{ Rendement } & \multicolumn{2}{l}{ Biomasse } & \multicolumn{2}{l}{ Indice de récolte } \\
\cline { 3 - 9 } Effects & \multirow{2}{*}{ Df } & Chisq & Pr (>Chisq) & Chisq & $\begin{array}{l}\text { Pr } \\
\text { (>Chisq) }\end{array}$ & Chisq & Pr (>Chisq) \\
\hline Fumure & 4 & 6.254 & 0.181 & 6.050 & 0.195 & 10.866 & $0.028^{*}$ \\
Campagne & 1 & 3.793 & 0.051 & 0.312 & 0.576 & 4.727 & $0.030^{*}$ \\
Fumure : Campagne & 4 & 3.349 & 0.501 & 5.462 & 0.243 & 15.357 & $0.004^{* *}$ \\
\hline
\end{tabular}

*significativité à un seuil $(\operatorname{Pr}<0.05)$

**significativité à un seuil $(\operatorname{Pr}<0.005)$ 
Une analyse séparée de chaque environnement est faite pour déterminer les variations de l'indice de récolte selon les traitements d'engrais. Une différence significative est observée seulement pendant la première campagne de blé à Sylla (Tableau 4). Le test de comparaison multiple a permis de déterminer l'effet de chaque traitement de fumure sur la variation de l'indice de récolte pendant la première campagne à
Sylla. La plus petite différence significative (PPDS) entre les différents traitements est de 10,3\%. La figure 2 montre la variation de l'indice de récolte pour les différents apports d'engrais minéral pendant la première campagne de blé à Sylla. En effet les traitements T3, T4 et T5 sont significativement différents du traitement $\mathrm{T} 1$.

Tableau 4 : L'analyse de variance des effets de traitements de fumure sur l'indice de récolte.

\begin{tabular}{l|l|l|l|l|l}
\hline \multirow{2}{*}{ Effects } & \multirow{2}{*}{} & \multicolumn{2}{|l|}{ Première campagne (Sylla) } & \multicolumn{2}{l}{ Deuxième campagne (Dara } \\
\cline { 2 - 6 } Bloc & Df & Sum Sq & $\operatorname{Pr}(>$ F) & Sum Sq. & $\operatorname{Pr}(>\mathbf{F})$ \\
Fumure & 3 & 749.75 & $0.012639^{*}$ & 210.95 & 0.2407129 \\
Résiduels & 4 & 830.8 & $0.017326^{*}$ & 334.8 & 0.1740513 \\
\hline
\end{tabular}

*significativité à un seuil $(\operatorname{Pr}<0.05)$

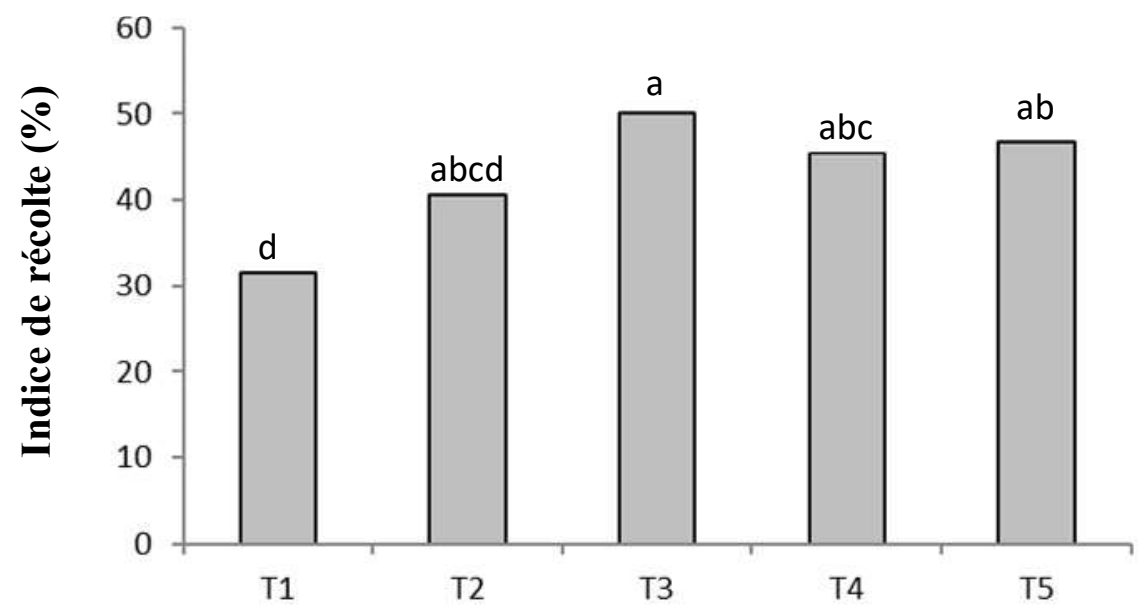

Figure 2: Effets des différents niveaux de fertilisation minérale sur l'indice de récolte du blé.

$N B$ : Deux traitements ayant une lettre en commun ne sont pas significativement différents

\section{$5 \quad$ DISCUSSION}

Les stations expérimentales de Dara et de Sylla ont été choisies pour représenter la diversité agroécologique présente dans la vallée du fleuve Sénégal, avec un accent particulier sur la moyenne vallée. Avec la disponibilité de l'eau et l'aménagement des périmètres irrigués dans la vallée du Fleuve Sénégal, le riz y est intensivement cultivé en monoculture, avec deux saisons consécutives (la saison chaude humide et la saison chaude sèche). Depuis quelques années la possibilité de la culture du blé dans ces rizières lais- sées en jachère pendant la saison froide est démontrée par les Chercheurs (Sall et al. 2018). Le développement d'un système de culture riz-riz-blé pour une agriculture intensive entraîne à la longue la dégradation des sols et l'appauvrissement en éléments nutritifs menaçant ainsi la productivité agricole (Vanlauwe et al. 2002). Un tel système de culture nécessite le cadrage du calendrier cultural accompagné de bonnes pratiques agricoles notamment l'utilisation d'une fertilisation raisonnée pour une agriculture durable 
car les pratiques agricoles vont entrainer la nécessité de restaurer les terres cultivées à travers l'utilisation des engrais chimiques. Pour les pays en développement, les engrais chimiques représentent une dépense importante et donc pour chaque culture, les agronomes doivent être en mesure de déterminer la quantité et le type d'élément nutritif les plus appropriés ainsi que le mode et la période d'application qui conviennent le mieux, compte tenu des caractéristiques du sol et des conditions climatiques propres à chaque région. Dans cette étude l'effet de différentes combinaisons d'élément nutritif des engrais chimiques sur la performance du riz et du blé a été évalué dans le but de proposer aux paysans de la vallée la meilleure formule possible. Quatre combinaisons d'élément nutritif $(\mathrm{N}, \mathrm{P}$ et $\mathrm{K})$ ont été appliquées sur le riz et le blé en comparaison avec le témoin absolu (aucune application d'engrais). Une différence significative entre les cinq traitements sur la performance des variétés de riz a été observée sur les trois caractères agronomiques étudiés (rendement grain, biomasse aérienne et indice de récolte). Le test de comparaison multiple a permis de constater que l'application de l'azote $(\mathrm{N})$ seule donne des rendements significativement supérieur au témoin absolu montrant la nécessité d'application des engrais chimique contenant l'azote pour booster la production rizicole. Ceci corrobore les résultats de Bado et al. (2010) qui indiquent qu'il faudra mettre le $\mathrm{N}$ chaque saison pour maintenir les rendements élevés du riz. Toutefois l'application de $\mathrm{N}$ seulement pourrait diminuer le carbone organique du sol Bado et al. (2010). Les faibles rendements du traitement témoin et celui associant $\mathrm{P}$ et $\mathrm{K}$ seulement peuvent être expliqués par la faible fertilité du sol notamment la carence en $\mathrm{N}$ disponible pour les plantes. La combinaison de l'Azote $(\mathrm{N})$ et du Phosphore $(\mathrm{P})$ donne un rendement grain et une biomasse aérienne supérieure à l'application de l'Azote seule ou son association avec le phosphore et le Potassium (K). Le faible niveau de $\mathrm{P}$ extractible dans le sol explique la nécessité d'épandage d'engrais $P$ pour dépasser la limite critique

\section{CONCLUSION}

Dans le contexte actuel de l'intensification de la production, il est important de développer des stratégies de gestion des engrais chimiques pour améliorer la productivité et la rentabilité du riz et du blé irrigué. C'est pourquoi une combinaison de tests a été réalisée de manière à déterminer la meilleure technique culturale transférable aux paysans. Les résultats montrent que pour le riz, si l'application de $\mathrm{N}$ est nécessaire pour augmenter la production de chaque saison, ce de $\mathrm{P}$ dans le sol et satisfaire le besoin du riz (Bado et al., 2008). En général, la plupart des terres irriguées dans les vallées de la savane sahélienne et soudanaise ont un sol avec des réserves considérable de $\mathrm{K}$ (Buri et al., 1999 ; Wopereis et al., 1999 ; Haefele et al., 2004). Ceci pourrait expliquer l'absence d'une réaction significative des plantes selon qu'on applique ou pas de K. La biomasse aérienne joue un rôle important en faveur du rendement grain (Mekhlouf et Bouzerzour, 2000 ; Abbassene et al., 1997 ; Masoni et al., 2007 ; Bahlouli et al., 2008) et constitue alors un paramètre important à considérer pour l'amélioration du rendement grain. Pour le blé, La différence significative entre les traitements eux même et entres les environnements (Sylla et Dara) est notée seulement pour l'indice de récolte. Il existe une importante interaction entre les traitements et les différents environnements concernant l'indice de récolte. L'analyse par environnement montre que c'est au niveau de Sylla seulement que cette différence entre les traitements existe sur l'indice de récolte. L'indice de récolte est un rapport exprimé en pourcentage, entre le poids des grains récolté et la biomasse aérienne sèche. Donc la variation de cet indice impacte sur le rendement grain et varie considérablement selon les espèces et variétés. Chez le blé les variétés semi-naines ont un indice de 0,47 , contre 0,40 chez les cultivars standard, tandis que chez le riz, pour les cultivars à haut rendement, l'indice varie de 0,23 à 0,50 . Les résultats obtenus sur le blé confirment ceux obtenus sur le riz. En effet, la combinaison de l'Azote $(\mathrm{N})$ et du Phosphore (P) donne un indice de récolte plus élevé par rapport à l'application de l'Azote seule ou son association avec le phosphore et le Potassium (K). Par ailleurs on note que l'association de $\mathrm{P}$ et $\mathrm{K}$ sans $\mathrm{N}$ peut augmenter significativement l'indice de récolte chez le blé. Ceci suggère que l'épandage de l'engrais $\mathrm{K}$ est essentiel chez le blé et donc l'utilisation des engrais NPK est fortement recommandée pour la culture du blé dans la vallée du fleuve Sénégal.

n'est probablement pas le cas pour $\mathrm{P}$ et $\mathrm{K}$. Par exemple, les applications saisonnières de $\mathrm{N}$ (chaque saison de culture) et des applications annuelles (une saison par an) de $\mathrm{P}$ et de $\mathrm{K}$ pourraient être une option pour améliorer la gestion des engrais, la production et la rentabilité du riz. Par contre la culture du blé nécessite l'application saisonnière de $\mathrm{N}, \mathrm{P}$ et $\mathrm{K}$ pour optimiser la production. Toutefois, les résultats de cette étude devraient être confirmés par d'autres 
travaux de fertilisation minérale en mettant un accent particulier sur le précédent culturale et l'analyse des

\section{REMERCIEMENTS}

Nous voudrions remercier le Conseil suédois de la recherche (Vetenskapsrådet) qui a financé la recherche le long du fleuve Sénégal par le biais du projet U-forsk 2013-2017 « Deployment of molecular

\section{RÉFÉRENCES}

Abbassenne F, Bouzerzour H. and Hachemi L : 1997. Phénologie et production du blé dur (Triticum durum Desf.) en zone semi-aride. Annales Agronomiques Institut National Agronomique 18 : 24-36.

Bado VB, Aw A. and Ndiaye M : 2010. Long-term effect of continuous cropping of irrigated rice on soil and yield trends in the Sahel of West-Africa. Nutrient Cycling in Agroecosystems $88: 133-141$.

Bado BV, DeVries ME, Haefele SM, Wopereis MCS. and Ndiaye MK: 2008. Critical limit of extractable phosphorous in a Gleysol for rice production in the Senegal River valley of west Africa. Soil Science and Plant Analysis 39: 202-206.

Bahlouli F, Bouzerzour H. and Benmahammed A : 2008. Effets de la vitesse et de la durée du remplissage du grain ainsi que de l'accumulation des assimilât de la tige dans l'élaboration du rendement du blé dur (Triticum durum Desf.) dans les conditions de culture des hautes plaines orientales d'Algérie. Biotechnology Agronomy Society and Environment $12: 31-39$.

Boy J. and Wilcke W : 2008. Tropical Andean forest derives calcium and magnesium from Saharan dust. Global Biogeochemical Cycles 22 : GB1027.

Buri MM, Ishida F, Kubota D, Masunaga T. and Wakatsuki T : 1999. Soils of flood plains of west Africa: General fertility status. Soil Science \& Plant Nutrition 45 : 37-50.

Dingkuhn M. and Sow A : 1997. Potential yields of irrigated rice in the Sahel. Miezan KM, Wopereis MCS, Dingkuhn M, Deckers J. and Randolph TF (Editors), West Africa Rice Development Association, Bouaké, Côte d'Ivoire.

Haefele SM, Wopereis MCS, Schloebohm AM. and Wiechmann H : 2004. Long-term fertility experiments for irrigated rice in the west Afri- éléments nutritifs du sol avant chaque campagne.

durum breeding to the Senegal basin : capacity building to face global warming ». Nous remercions en particulier Dr. Filippo Bassi de ICARDA qui a accompagné et facilité la réalisation de ces travaux.

can Sahel : Effects on soil characteristics. Field Crops Research 83 : 61-77.

Ladha JK, Fischer KS, Hosssain M., Hobbs PR. And Hardy B : 2000. Improving the productivity and sustainability of rice-wheat systeme of the Indo-Gangetic Plains : a synthesis of NARS-IRRI partnership research. International Rice Research Institute $40: 1-31$.

Masoni A, Ercoli L, Mariotti M. and Arduini I : 2007. Post-anthesis accumulation and remobilization of dry matter, nitrogen and phosphorus in durum wheat as affected by soil type. European Journal of Agronomy 26 : 179-186.

Mekhlouf A. and Bouzerzour H : 2000. Déterminisme génétique et associations entre le rendement et quelques caractères à variation continue chez le blé dur (Triticum durum Desf.). Recherche Agronomique 7 : 37-49.

Sall AT, Kabbaj H, Cisse M, Gueye H, Ndoye I, Maltouf AF, El-Mourid M, Ortiz R. and Bassi FM : 2018. Heat tolerance of durum wheat (tritcum durum desf.) elite germplasm tested along the Senegal River. Jounal of Agricultural Science $10: 217$.

Tilman D, Cassman KG, Matson PA, Naylor R. and Polasky S : 2002. Agricultural sustainability and intensive production practices. Nature 418: 671-677

Bernard V, Palm C, Murwira H. and Merckx R : 2002. Organic resource management in subSaharan Africa: validation of a residue quality-driven decision support system. Agronomy, EDP Sciences $22: 839-846$.

Wopereis MCS, Donovan C, Nebié B, Guindo D. and Ndiaye MK : 1999. Soil fertility management in irrigated rice systems in the Sahel savanna region of West Africa. Field Crops Research 61 : 125-145. 